\title{
Disentangling magnetic order on nanostructured surfaces
}

\author{
D. Erb,,${ }^{1, *}$ K. Schlage,${ }^{1}$ L. Bocklage, ${ }^{1,2}$ R. Hübner, ${ }^{3}$ D. G. Merkel, ${ }^{4, \dagger}$ R. Rüffer, ${ }^{4}$ H.-C. Wille, ${ }^{1}$ and R. Röhlsberger ${ }^{1,2}$ \\ ${ }^{1}$ Deutsches Elektronen-Synchrotron DESY, Notkestraße 85, 22607 Hamburg, Germany \\ ${ }^{2}$ The Hamburg Centre for Ultrafast Imaging, Luruper Chaussee 149, 22761 Hamburg, Germany \\ ${ }^{3}$ Helmholtz-Zentrum Dresden-Rossendorf, Bautzner Landstraße 400, 01328 Dresden, Germany \\ ${ }^{4}$ European Synchrotron Radiation Facility, 71 avenue des Martyrs, CS 40220, 38043 Grenoble Cedex 9, France
}

(Received 27 April 2017; published 17 July 2017)

\begin{abstract}
We present a synchrotron-based x-ray scattering technique which allows disentangling magnetic properties of heterogeneous systems with nanopatterned surfaces. This technique combines the nanometer-range spatial resolution of surface morphology features provided by grazing incidence small angle x-ray scattering and the high sensitivity of nuclear resonant scattering to magnetic order. A single experiment thus allows attributing magnetic properties to structural features of the sample; chemical and structural properties may be correlated analogously. We demonstrate how this technique shows the correlation between structural growth and evolution of magnetic properties for the case of a remarkable magnetization reversal in a structurally and magnetically nanopatterned sample system.
\end{abstract}

DOI: 10.1103/PhysRevMaterials.1.023001

Knowledge of the relations between morphology and physical properties of nanometer-scaled objects is key to engineer the functionalities of devices in nanotechnology. Prominent examples are the size- and shape-dependent magnetic properties of nanoparticles [1,2], which are highly relevant for medical diagnostics and therapy, and ferromagnetic components in magnonic devices [3] and magnetoplasmonic systems [4], or the size- and composition-dependent reactivity and morphological changes of catalytically active nanoparticles during chemical reaction $[5,6]$. Thus methods yielding the nanostructure morphology with high spatial resolution and enabling comprehensive chemical characterization or delivering precise information on magnetic properties are indispensable tools in nanoscience: Physicochemical characterization is routinely accomplished by methods such as high-resolution transmission electron microscopy [7], grazing incidence small angle $\mathrm{x}$-ray scattering [8], mass spectroscopy [9], dynamic light scattering [10], surface plasmon resonance [11], or spectroscopic methods using radiation from infrared to x-ray wavelengths [7-14]. For magnetic nanostructure characterization, a variety of techniques has been established, all with unique assets but also with certain drawbacks. Scanning probe techniques provide very high spatial resolution [15], but are insensitive to magnetization dynamics. Kerr and Faraday microscopy offer picosecond time resolution, but their spatial resolution is merely in the submicrometer range [16]. Scanning electron microscopy with polarization analysis measures the magnetization vector orientation directly via the spin polarization of secondary electrons [17] and x-ray photoemission electron microscopy combines good spatial and temporal resolution with element specificity [18]. Being based on the detection of secondary electrons, however, both require ultrahigh vacuum conditions and only allow for applying very weak or localized magnetic

\footnotetext{
*Present address: Helmholtz-Zentrum Dresden-Rossendorf, Bautzner Landstraße 400, 01328 Dresden, Germany; d.erb@hzdr.de

${ }^{\dagger}$ On leave from Wigner Research Centre for Physics, Hungarian Academy of Sciences, H-1525 Budapest, Hungary.
}

fields to the sample. Diffraction magneto-optical Kerr effect measurements are simple to realize and can accommodate various sample environments [19], but the wavelength of the employed light makes nanostructure characterization unfeasible. Neutron scattering techniques can provide both structural and magnetic information [20], but suffer from the low neutron fluxes, which limits the possibilities for in situ measurements, e.g., during nanostructure growth. Methods based on x-ray transmission or scattering in transmission geometry (scanning transmission $\mathrm{x}$-ray microscopy, transmission imaging $\mathrm{x}$-ray microscopy, or x-ray holography) offer element-specificity and high spatial resolution, but pose constraints on sample environment volumes, require a transmissive substrate, or demand the sample to be processed by microfabrication techniques [21,22].

We propose a method for investigating nanometer-scaled objects, which can simultaneously deliver morphological parameters with subnanometer lateral precision and the static and dynamic magnetic characteristics of these structures under in situ conditions of growth, high temperatures, reactive environments, or strong magnetic fields. To achieve this, we combine two $\mathrm{x}$-ray scattering techniques into a single experiment, namely grazing incidence small-angle $\mathrm{x}$-ray scattering (GISAXS) and nuclear resonant scattering (NRS). GISAXS provides morphological characterization of nanometer-sized surface features, based on the angular distribution of scattered photons depending on the sample structure. Thus threedimensional shapes and lateral arrangements of nanostructures supported on surfaces or buried in thin films are obtained with nanometer resolution [23]. NRS yields information on magnetic ordering, enables precise determination of in-plane and out-of-plane magnetic moment orientation and allows for sensitive detection of magnetization dynamics with an accuracy of a few degrees and submicrosecond time resolution [24-27] by probing the coherent elastic resonant scattering of photons from Mössbauer-active nuclei [28]. Intensity maxima in a GISAXS pattern originate from photons, which are scattered off different periodically repeated structural components of the sample. Photons, which have been resonantly scattered from nuclei, are identified by their time delay with respect to the 
photons, which have been nonresonantly scattered from electrons. The coherent elastic nuclear resonant scattering of photons results in a characteristic time spectrum of the detected intensity. At the specular intensity maximum, this time spectrum reflects the integrated magnetic properties of the entire sample [29,30]. By placing the detector at selected off-specular intensity maxima within the pattern, however, one obtains information on the magnetic properties of the specific structural component of the sample, which the selected intensity maximum is related to. Thus both structural information and sitespecific magnetic characteristics are gathered simultaneously, directly revealing the correlations between these properties.

From merging GISAXS and NRS into a single technique referred to as GINSAXS, comprehensive structural and magnetic information on heterogeneous systems with periodic nanoscopic surface morphology can be obtained. In this paper, we provide an experimental proof of principle for the aforementioned concept under two different in situ conditions. While we apply GINSAXS to disentangle heterogeneous magnetic properties, the method could also be employed to elucidate heterogeneous chemical composition or crystal structure: the hyperfine interactions probed by NRS also characterize local chemical environments and local lattice structures of resonant nuclei [31-33]. Generally, a combination of GISAXS with NRS is applicable to samples with nonplanar surface or interface morphologies, such as periodic arrangements of uniform nanostructures with tilted or curved surfaces. Such a technique could be highly beneficial for studying facet-selective adsorption [34,35], overgrowth [36-38], or reactivity [39,40]. It could also help to clarify the development of magnetic order and the magnetization reversal in faceted nanoparticles with magnetic shells [41], or serve to study the magnetic properties of nanoparticle-based mesocrystals [42-44].

We demonstrate the capability of GINSAXS by investigating a nanostructured sample system with periodically varying morphological and magnetic properties under in situ conditions requiring ultrahigh vacuum and external magnetic fields, respectively. The sample consists of an $\alpha-\mathrm{Al}_{2} \mathrm{O}_{3}$ substrate with parallel nanometer-scale facets (see Refs. [45,46]), supporting a thin continuous Fe film. The substrate has an average facet height of $h=15 \mathrm{~nm}$ and period of $L=80 \mathrm{~nm}$, the average facet tilt angles are $\beta_{R}=30^{\circ}$ and $\beta_{S}=17^{\circ}$. From these values, average facet widths of $w_{R} \approx 32 \mathrm{~nm}$ and $w_{S} \approx 55 \mathrm{~nm}$ are calculated. The Fe film is grown by room-temperature sputter deposition from a polar angle of $45^{\circ}$ and an azimuthal angle of $90^{\circ}$ with respect to the facet edges. Here, the R-plane facets are facing the sputtering source, so that the deposition rate is higher on these facets than on the S-plane facet, which are avert from the source. Consequently, the Fe film consists of thicker regions $(18 \mathrm{~nm})$ on the narrower $\mathrm{R}$-plane facets and thinner regions $(13 \mathrm{~nm})$ on the wider S-plane facets [see Fig. 1(a)]. As determined from a GISAXS pattern recorded with the facet edges aligned perpendicular to the incident x-ray beam, the Fe film is polycrystalline with a crystallite size of approximately $5 \mathrm{~nm}$ (see Ref. [45]). To prevent oxidation of the Fe film, the sample was capped with a $\mathrm{Cr}$ layer. The corrugated shape of the Fe film induces a uniaxial magnetic anisotropy with the easy axis of magnetization parallel to the substrate facet edges [47].

As summarized in Fig. 1, GINSAXS is conducted in the following sequence. First, a conventional 2D GISAXS pattern (c) time spectrum

(b) 2D scattering pattern $a_{\mathrm{f}}(\mathrm{deg})$

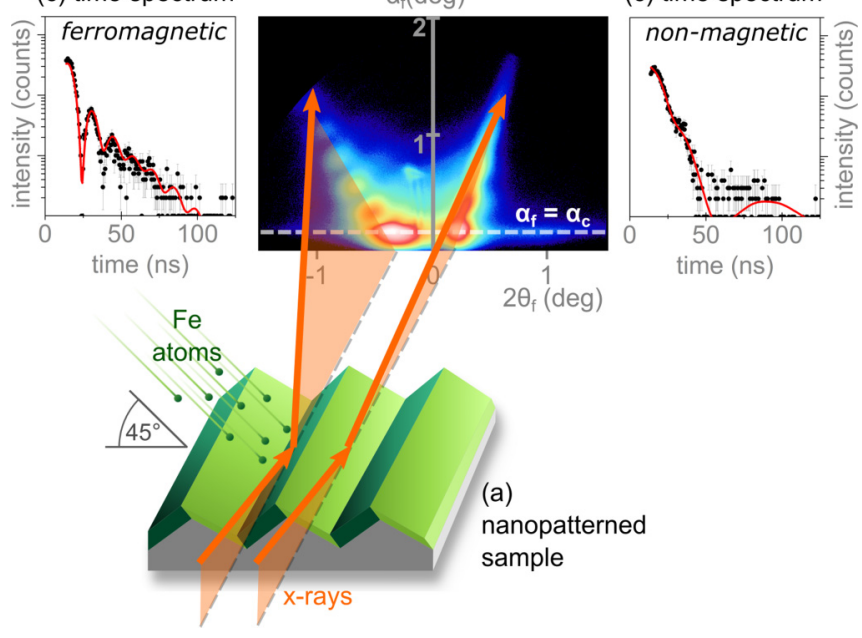

FIG. 1. Illustration of the GINSAXS principle by means of a sample with faceted surface: (a) A heterogeneous Fe film is grown on a nanofaceted substrate by sputter deposition under non-normal incidence. (b) The 2D scattering pattern is the same for both nonresonantly and resonantly scattered photons; photons detected in the left (right) crystal truncation rod (CTR) carry information on the Fe film regions on the R-plane (S-plane) facets. (c) Using a time-resolving detector to record only resonant photons, nuclear resonant time spectra are taken at the left and right CTR, evidencing the different magnetic properties of the Fe film on the R-plane and S-plane facets, respectively.

is recorded at a suitable angle of incidence using an area detector. With the facet edges aligned parallel to the incident $\mathrm{X}$-ray beam, the GISAXS pattern is characterized by two crystal truncation rods (CTRs) originating from the R-plane and S-plane facets, respectively [8]. Second, the positions of intensity maxima, which are specific for certain structural units of the sample are selected from the GISAXS pattern. Here, these are the positions of highest intensity along the two CTRs. The angle of incidence is adjusted to maximize the resonantly scattered intensity. Third, using a time-resolving point detector (avalanche photo diode, APD) nuclear resonant time spectra are recorded at the selected positions. The angular distribution of scattered intensity measured in the GISAXS pattern is a signature of the nanometer-scale surface morphology of the sample: the two tilted scattering rods correspond to the Fe film regions supported by the substrate facets with R-plane and S-plane orientation, respectively. Intensity modulations along the CTRs are related to the Fe film thicknesses on the respective facet surfaces (similar to Kiessig fringes). The film thicknesses and geometrical parameters describing the sample morphology are obtained by simulating the GISAXS patterns using the program FitGISAXS [48]. The nuclear resonant time spectra serve as fingerprints of the magnetic characteristics of the different repeat units of the sample, conveying information on the degree of magnetic order and the magnetization orientation. The shape of a time spectrum correlates with these properties via strength and orientation, respectively, of the magnetic hyperfine field $\mathbf{B}_{h f}$ at the Fe nuclei. Time spectra were fitted using the program CONUSS [49] (see Supplemental Material [45]). 
(a)

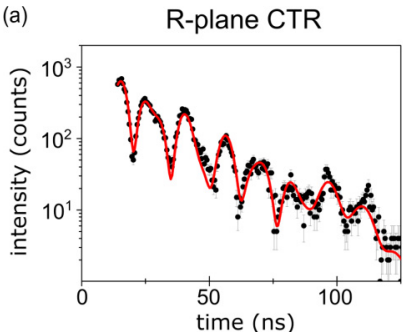

(b)

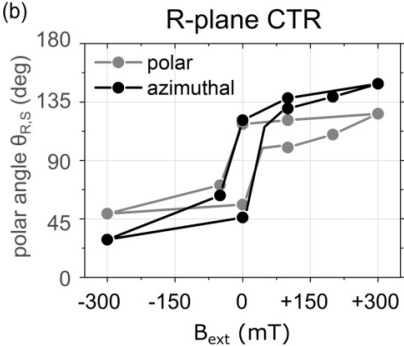

(c)

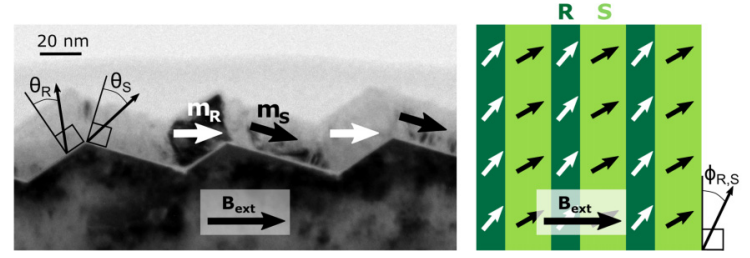

FIG. 2. (a) Exemplary NRS time spectra and corresponding fits (red) for the Fe film regions on R- and S-plane facets, respectively, in an applied magnetic field of $B_{\text {ext }}=200 \mathrm{mT}$. The differences in the beat patterns evidence unequal magnetization orientations. (b) Azimuthal (in-plane) and polar (out-of-plane) magnetization orientation in R-plane and S-plane film regions as extracted from fits of the NRS time spectra. Solid lines are guides to the eye. (c) A cross-sectional transmission electron micrograph (left) and a top-view sketch (right) of the sample, with arrows indicating the out-of-plane and in-plane components of the magnetization vectors relative to external magnetic field.

Following the procedure described above, GINSAXS was performed at an x-ray energy of $14.4 \mathrm{keV}$, i.e., the resonance energy of ${ }^{57} \mathrm{Fe}$, at the high resolution dynamics beamline P01 at PETRA III and the nuclear resonance beamline ID18 at ESRF (see Ref. [45]). We performed two independent in situ GINSAXS experiments on a sample system which is structurally and magnetically heterogeneous on the nanoscale. The experiments provide spatially resolved information on the magnetization reversal and allow correlating film growth and development of ferromagnetic ordering.

In the first experiment, we resolve the heterogeneous magnetization reversal in the nanostructured Fe film upon applying an external magnetic field. The polar and azimuthal angles $\theta_{R, S}$ and $\phi_{R, S}$ of the magnetization as extracted from fitting the NRS time spectra for both R-plane and S-plane film regions are plotted as functions of the external magnetic field in Fig. 2(b). The angles are defined independently for R-plane and $\mathrm{S}$-plane film regions, such that the magnetization is in the plane of the respective film region for a polar angle of $\theta_{R, S}=90^{\circ}$ and parallel to the facet edges for an azimuthal angle of $\phi_{R, S}=0^{\circ}$. The hysteretic behavior of the azimuthal magnetization orientation is similar for the R-plane and S-plane regions of the Fe film: the magnetization is displaced from the easy axis parallel to the facet edges to similar extents but does not align fully with the orientation of the external magnetic field. This can be accounted for by the pronounced uniaxial in-plane magnetic anisotropy of the uniaxially corrugated film. The R-plane and S-plane regions of the Fe film differ markedly, however, in the polar magnetization orientation [47]; in the $\mathrm{S}$-plane $\mathrm{Fe}$ film regions the polar magnetization orientation remains almost constant at $\theta_{S}=90^{\circ}$, i.e., it remains parallel to the film plane of these regions even at highest field strength. In contrast, the magnetization in the R-plane regions is deflected to an orientation in between the direction of the external magnetic field at $\theta=60^{\circ}$ and $120^{\circ}$, respectively, and the magnetization in the S-plane film regions at $\theta=43^{\circ}$ and $137^{\circ}$, respectively. The film regions on the $\mathrm{S}$-plane facets are both thinner and wider than those on the R-plane facets. Thus the shape anisotropy is more pronounced and in-plane orientation of the magnetization is preferred in the S-plane film regions. Furthermore, the external magnetic field is applied parallel to the average sample surface and does not enclose the same angle with the R-plane and S-plane facets. Consequently, the magnitude of the external magnetic field component normal to the film plane is by approximately $70 \%$ larger for the $\mathrm{R}$-plane film regions on the R-plane facets. Interface coupling between the $\mathrm{Fe}$ film and the antiferromagnetic $\mathrm{Cr}$ capping layer with high magnetic anisotropy may be a cause for the inertness observed in the magnetization returning to its easy axis orientation at remanence. The influence of this effect on the measurement is strong due to the surface sensitivity of NRS at $\alpha_{i}=0.16^{\circ}$. NRS spectra taken at higher incidence angles indicate a spring-like magnetization structure of the $\mathrm{Fe}$ film, where the top layers of the Fe film are coupled to the $\mathrm{Cr}$ capping layer, while the bottom layers are free to relax toward the easy axis orientation when no external field is applied (see Ref. [45]).

Furthermore, we observed the correlation of growth and magnetic stabilization in the stripelike regions of the Fe film in an in situ experiment during Fe deposition. Exemplary GISAXS patterns taken during growth of the Fe film are compared to the corresponding simulations in Fig. 3 (see Ref. [45]). The periods of intensity modulation along the CTRs decrease in correspondence to the increasing film thicknesses; the unequal modulation periods for each deposition stage evidence the different thicknesses of the Fe film on S-plane and R-plane facets. Simultaneously to the film growth observed in the GISAXS patterns, the evolution of magnetic properties is evidenced by characteristic changes in the time spectra. Figure 4 depicts sequences of time spectra for the film regions on R-plane and S-plane facets recorded during growth and sketches the corresponding strength and orientation of magnetic moments. A nonmagnetic state is characterized by the lack of a beat pattern on the time spectrum, while the time spectrum of a ferromagnetic state exhibits a pronounced beat pattern. The sequences show the successive evolution of beat patterns in the time spectra for the different film regions, thus evidencing a consecutive transition from a nonmagnetic to a ferromagnetic state first in the R-plane film regions, then in S-plane regions. Notably, a state is observed in which the S-plane film regions are still nonmagnetic, while the 

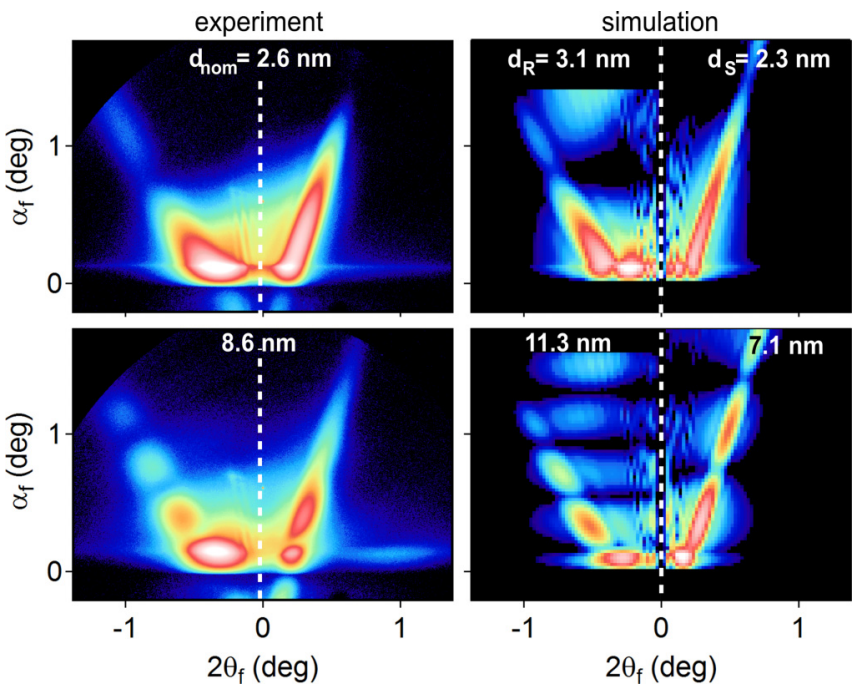

FIG. 3. Exemplary nonresonant GISAXS patterns and corresponding simulations for two stages of Fe deposition onto the faceted substrate. The dashed lines indicate the specular scattering plane. The GISAXS patterns are dominated by the tilted crystal truncation rods (CTRs) originating from the surface facets. The different periods of the intensity modulations along the CTRs result from the different thicknesses of the Fe film on the R-plane and S-plane facets, respectively. Labels state the nominally deposited Fe film thickness $d_{\text {nom }}$ and the thicknesses of the film regions $d_{R}$ and $d_{S}$ as obtained from simulations.

R-plane regions already exhibit a magnetization parallel to the facet edges. Full ferromagnetic order is observed at a film thickness of $(2.8 \pm 0.1) \mathrm{nm}$ for the R-plane Fe film regions. In the S-plane regions, ferromagnetic order is established at a thickness of $(2.3 \pm 0.1) \mathrm{nm}$ already, which may be due to the direct contact with the fully ferromagnetic R-plane film regions. In polycrystalline Fe thin films with uniaxially corrugated shape, ferromagnetic ordering was observed at film thicknesses $1 \mathrm{~nm}<d<3 \mathrm{~nm}$; the results of this experiment thus agree well with former findings [50]. For thicknesses larger than $2.8 \mathrm{~nm}$ and $2.3 \mathrm{~nm}$, respectively, all film regions on the R-plane facets have a magnetic hyperfine field magnitude close to the $\alpha$-Fe bulk value. Both the R-plane and S-plane film regions now show time spectra with pronounced beat patterns evidencing ferromagnetically ordered $\mathrm{Fe}$ with the magnetization oriented exactly parallel to the direction of the incoming beam, i.e., parallel to the facet edges. Cross-sectional transmission electron microscopy [see Fig. 2(c)] confirms that the free surface of the Fe film and its interface with the substrate are parallel as indicated by the sharp intensity modulations in the GISAXS patterns.

In conclusion, GINSAXS, a combination of GISAXS and NRS, allows detecting the magnetic information from different

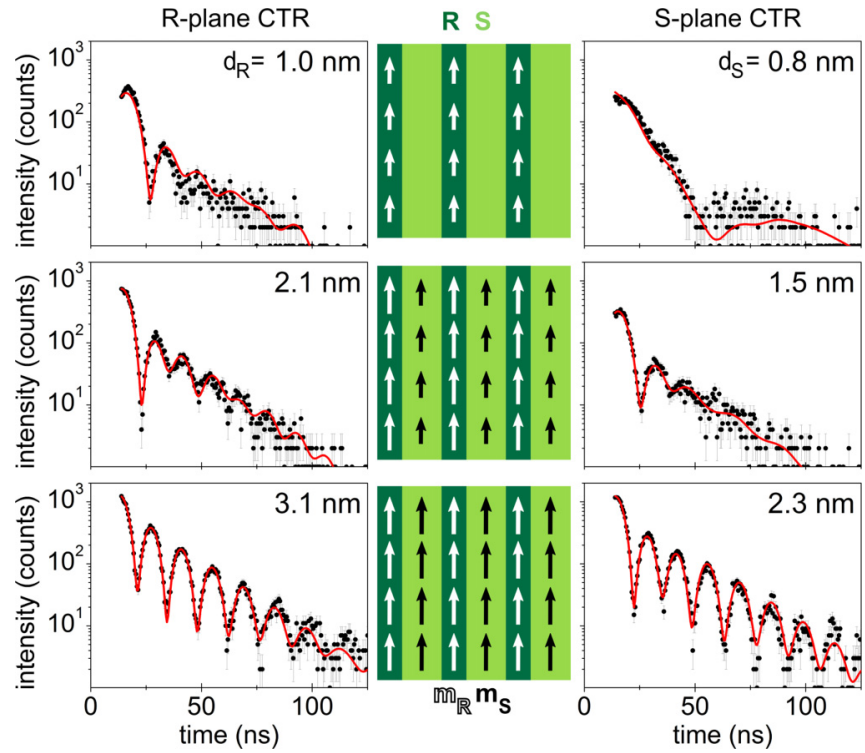

FIG. 4. Sequences of NRS time spectra (top to bottom) with corresponding fits (red) for film regions on R-plane and S-plane facets at subsequent stages of Fe deposition. The development of the characteristic beat pattern evidences the successive transition from a nonmagnetic to a ferromagnetic state in the R-plane and S-plane regions. Strength and orientation of magnetic moments in the film regions on R-plane and S-plane facets are indicated by black and white arrows, respectively.

structural units of a nanostructured sample separately. This approach has a great potential for characterizing corrugated magnetic materials, which are of interest for applications in magnetic sensing due to their shape-induced uniaxial magnetic anisotropy. Also standing spin waves in magnetic lattices may be investigated. Furthermore, it is conceivable to employ it for studying supported or buried nanoparticles with faceted or curved surfaces or with core-shell morphologies. The successful demonstration of GINSAXS may also encourage testing the feasibility of combining X-ray absorption near edge structure (XANES) and GISAXS in an analogous manner: this would be a powerful tool for investigating nanostructures with shape-dependent heterogeneous chemical properties, for instance, for in situ studies of catalytic processes with facetselective reactivity.

We gratefully acknowledge David Babonneau, Université de Poitiers, for implementing a new form factor in FitGISAXS on our request, which enabled simulating the GISAXS patterns of the $\mathrm{Fe}$ film on the faceted substrate. We thank Shengqiang Zhou, Helmholtz-Zentrum Dresden-Rossendorf, for measuring VSM hysteresis curves of the sample.
[1] B. Mehdaoui, A. Meffre, J. Carrey, S. Lachaize, L.-M. Lacroix, M. Gougeon, B. Chaudret, and M. Respaud, Adv. Funct. Mater. 21, 4573 (2011).

[2] L. H. Reddy, J. L. Arias, J. Nicolas, and P. Couvreur, Chem. Rev. 112, 5818 (2012).
[3] J. Topp, D. Heitmann, M. P. Kostylev, and D. Grundler, Phys. Rev. Lett. 104, 207205 (2010).

[4] G. Armelles, A. Cebollada, A. García-Martín, and M. U. González, Adv. Optical Mater. 1, 10 (2013). 
[5] K. Wettergren, F. F. Schweinberger, D. Deiana, C. J. Ridge, A. S. Crampton, M. D. Rötzer, T. W. Hansen, V. P. Zhdanov, U. Heiz, and C. Langhammer, Nano Lett. 14, 5803 (2014).

[6] U. Hejral, P. Müller, O. Balmes, D. Pontoni, and A. Stierle, Nat. Commun. 7, 10964 (2016).

[7] D. Li, M. H. Nielsen, J. R. I. Lee, C. Frandsen, J. F. Banfield, and J. J. De Yoreo, Science 336, 1014 (2012).

[8] M. Rauscher, R. Paniago, H. Metzger, Z. Kovats, J. Domke, J. Peisl, H.-D. Pfannes, J. Schulze, and I. Eisele, J. Appl. Phys. 86, 6763 (1999).

[9] K. Park, D. Lee, A. Rai, D. Mukherjee, and M. R. Zachariah, J. Phys. Chem. B 109, 7290 (2005).

[10] R. Pecora, J. Nanopart. Res. 2, 123 (2000).

[11] V. Juvé, M. F. Cardinal, A. Lombardi, A. Crut, P. Maioli, J. Pérez-Juste, L. M. Liz-Marzán, N. Del Fatti, and F. Vallée, Nano Lett. 13, 2234 (2013).

[12] N. Shukla, C. Liu, P. M. Jones, and D. Weller, J. Magn. Magn. Mater. 266, 178 (2003).

[13] V. Amendola and M. Meneghetti, J. Phys. Chem. C 113, 4277 (2009).

[14] P. Prieto, V. Nistor, K. Nouneh, M. Oyama, M. Abd-Lefdil, and R. Díaz, Appl. Surf. Sci. 258, 8807 (2012).

[15] R. Wiesendanger, Rev. Mod. Phys. 81, 1495 (2009).

[16] J. McCord, J. Phys. D: Appl. Phys. 48, 333001 (2015).

[17] K. Koike, Microscopy 62, 177 (2013).

[18] X. Cheng and D. Keavney, Rep. Prog. Phys. 75, 026501 (2012).

[19] M. Grimsditch and P. Vavassori, J. Phys.: Condens. Matter 16, R275 (2004).

[20] H. Zabel, K. Theis-Bröhl, and B. P. Toperverg, Polarized neutron reflectivity and scattering from magnetic nanostructures and spintronic materials, in Handbook of Magnetism and Advanced Magnetic Materials (Wiley, New York, 2007).

[21] J. Stöhr, Y. Wu, B. Hermsmeier, M. Samant, G. Harp, S. Koranda, D. Dunham, and B. Tonner, Science 259, 658 (1993).

[22] J. J. Turner, X. Huang, O. Krupin, K. A. Seu, D. Parks, S. Kevan, E. Lima, K. Kisslinger, I. McNulty, R. Gambino, S. Mangin, S. Roy, and P. Fischer, Phys. Rev. Lett. 107, 033904 (2011).

[23] G. Renaud, R. Lazzari, and F. Leroy, Surf. Sci. Rep. 64, 255 (2009).

[24] R. Röhlsberger, Hyperfine Interact. 123/124, 455 (1999).

[25] R. Röhlsberger, J. Bansmann, V. Senz, K. L. Jonas, A. Bettac, K. H. Meiwes-Broer, and O. Leupold, Phys. Rev. B 67, 245412 (2003).

[26] K. Schlage and R. Röhlsberger, J. Elect. Spect. Rel. Phenom. 189, 187 (2013).

[27] L. Bocklage, C. Swoboda, K. Schlage, H.-C. Wille, L. Dzemiantsova, S. Bajt, G. Meier, and R. Röhlsberger, Phys. Rev. Lett. 114, 147601 (2015)

[28] R. Röhlsberger, Springer Tracts in Modern Physics, Vol. 208: Nuclear Condensed Matter Physics with Synchrotron Radiation (Springer, Berlin, 2004).
[29] K. Schlage, S. Couet, S. V. Roth, U. Vainio, R. Rüffer, M. M. A. Kashem, P. Müller-Buschbaum, and R. Röhlsberger, New J. Phys. 14, 043007 (2012).

[30] G. Sharma, A. Gupta, M. Gupta, K. Schlage, and H.-C. Wille, Phys. Rev. B 92, 224403 (2015).

[31] G. Vogl, E. Partyka-Jankowska, M. Zając, and A. I. Chumakov, Phys. Rev. B 80, 115406 (2009).

[32] V. Potapkin, C. McCammon, K. Glazyrin, A. Kantor, I. Kupenko, C. Prescher, R. Sinmyo, G. V. Smirnov, A. I. Chumakov, R. Rüffer, and L. Dubrovinski, Nat. Commun. 4, 1427 (2013).

[33] K. Freindl, E. Partyka-Jankowska, W. Karaś, M.Zając, E. Madej, N. Spiridis, M. Ślęzak, T. Ślęzak, D. Wiśnios, and J. Korecki, Surf. Sci. 617, 183 (2013)

[34] C.-Y. Chiu, H. Wu, Z. Yao, F. Zhou, H. Zhang, V. Ozolins, and Y. Huang, J. Am. Chem. Soc. 135, 15489 (2013).

[35] P. S. Mdluli, N. M. Sosibo, P. N. Mashazi, T. Nyokong, R. T. Tshikhudo, A. Skepu, and E. van der Lingen, J. Mol. Struct. 1004, 131 (2011).

[36] S. Sun, C. Kong, H. You, X. Song, B. Ding, and Z. Yang, Cryst. Eng. Comm. 14, 40 (2012).

[37] X.-W. Liu, Langmuir 27, 9100 (2011).

[38] M. N. Mankin, R. W. Day, R. Gao, Y.-S. No, S.-K. Kim, A. A. McClelland, D. C. Bell, H.-G. Park, and C. M. Lieber, Nano Lett. 15, 4776 (2015).

[39] Y.-W. Harn, T.-H. Yang, T.-Y. Tang, M.-C. Chen, and J.-M. Wu, Chem. Cat. Chem. 7, 80 (2015).

[40] R. Li, F. Zhang, D. Wang, J. Yang, M. Li, J. Zhu, X. Zhou, H. Han, and C. Li, Nat. Commun. 4, 1432 (2013).

[41] F. Nasirpouri, S. J. Bending, L. M. Peter, and H. Fangohr, Thin Solid Films 519, 8320 (2011).

[42] S. Disch, E. Wetterskog, R. P. Hermann, G. Salazar-Alvarez, P. Busch, T. Brückel, L. Bergström, and S. Kamali, Nano Lett. 11, 1651 (2011).

[43] C.-J. Chen, R.-K. Chiang, and S.-L. Wang, Cryst. Eng. Comm. 15, 9161 (2013).

[44] E. Josten, E. Wetterskog, A. Glavic, P. Boesecke, A. Feoktystov, E. Brauweiler-Reuters, U. Rücker, G. Salazar-Alvarez, T. Brückel, and L. Bergström, Sci. Rep. 7, 2808 (2017).

[45] See Supplemental Material at http://link.aps.org/supplemental/ 10.1103/PhysRevMaterials.1.023001 for details on: experimental realization, NRS and GISAXS analysis, magnetic hysteresis and depth dependence of magnetization orientation.

[46] J. R. Heffelfinger and C. B. Carter, Surf. Sci. 389, 188 (1997).

[47] M. O. Liedke, M. Körner, K. Lenz, M. Fritzsche, M. Ranjan, A. Keller, E. Čižmár, S. A. Zvyagin, S. Facsko, K. Potzger, J. Lindner, and J. Fassbender, Phys. Rev. B 87, 024424 (2013).

[48] D. Babonneau, J. Appl. Crystallogr. 43, 929 (2010).

[49] W. Sturhahn, Hyperfine Interact. 125, 149 (2000).

[50] Y. Shiratsuchi, Y. Endo, M. Yamamoto, D. Li, and S. D. Bader, J. Appl. Phys. 95, 6897 (2004). 